\title{
On Technological Aspects of Active Ageing
}

\author{
Hamideh Afsarmanesh \\ Computer Science Department \\ University of Amsterdam
}

Science Park 107, 1098 XG Amsterdam, The Netherlands

h.Afsarmanesh@uva.nl

\author{
Simon S. Msanjila \\ Information and Communication Technology Department \\ Mzumbe University \\ P. O. Box 1, Mzumbe-Morogoro, Tanzania \\ smsanjila@mzumbe.ac.tz
}

\author{
Luis M. Camarinha-Matos \\ Faculty of Sciences and Technology \& UNINOVA \\ Universidade Nova de Lisboa \\ 2829-516 Monte Caparica, Portugal \\ cam@uninova.pt
}

\begin{abstract}
The ePAL initiative aims at developing a roadmap for extension of professional active life of the retiring and retired knowledge workers in Europe. Three specific perspectives are identified in this roadmap, and defined to comprehensively address relevant dimensions of the ePAL environment. These include: (1) the technological perspective that addresses both the needed technology and the support services, (2) the societal perspective that addresses both the human factors and the societal aspects, and (3) the Organizational perspective that addresses both the economical managerial aspects and the governmental aspects of these environments. This paper however aims to zoom in on the technological perspective of the ePAL. It introduces the four main ICT research and development areas that are identified for strengthening and realization of active ageing. The technological perspective of the ePAL's vision constitutes a substantial part of the ePAL roadmap recommendation results. The paper further addresses the state of the art in each of these four areas and exemplifies their application needs in the ePAL environment.
\end{abstract}

Keywords- Collaborative networks, active ageing, Roadmap, Technical perspective of active ageing

\section{INTRODUCTION}

A critical challenge facing the European society is how to support the "active ageing / ageing well" [1] process for its knowledge workers in the coming decades. It is required to identify new societal positions, governmental policies, and organizational structures, together with the needed technology and support systems and tools to improve the existing and emerging situations. It is desired that the retired/retiring elderly citizens can remain partially involved in the market/society, and have the opportunity to use their knowledge and expertise in making valued contributions to the communities where they live. Consequently, it is foreseen that in near future retirement for knowledge workers will no longer represent the end of working period, rather a lifestyle and career transition, where the retirees in principle have multiple options - such as continuing to work (though perhaps at a different pace), returning to school for additional training or education, changing career, venturing into entrepreneurship, becoming more involved in volunteer work, and simply enjoying leisure and travel possibilities - thus a mix of working, learning, relaxing, and trying new things is being gradually foreseen as possibilities.

Currently most initiatives supporting seniors are focused on their socialization and entertainment activities, while new organizational forms and mechanisms are emerging, which are focused on providing ways to help senior professionals remain partially active after retirement. The ePAL roadmap aims at supporting senior professionals with an enjoyable active life, balanced between work and leisure, and as such its approach complements some other initiatives that target supporting the active ageing workers, e.g. the eSangathan project [2] and others, which investigates the use of ICT, the collaborative working environments and web 2.0 tools by senior professionals, and the role that can be played by training seniors for this endeavor. Some work also focuses on identification of the best fit existing ICT tools in the market that can be used by senior professionals to improve their continued work.

Within the ePAL roadmap three orthogonal perspectives are defined, called the technological, societal, and organizational perspectives, to comprehensively address this problem area and identify ePAL's future vision, as well as identifying the activities required to reach that vision [1].

In the technological sector, the main focus of this paper, it is clear that many of the present advances in ICT are not aimed at Senior Professionals as a target user [3]. This is perhaps because those that design the software by and large do not come from a senior generation themselves, and have no proper understanding of the needs of seniors, to consider them as input to their design. This however leads to a vicious cycle, whereby software and their applications are designed for a younger user who then becomes its chief consumer and

Permission to make digital or hard copies of all or part of this work for personal

or classroom use is granted without fee provided that copies are not made or

distributed for profit or commercial advantage and that copies bear this notice

and the full citation on the first page. To copy otherwise, to republish, to post on

servers or to redistribute to lists, requires prior specific permission and/or a fee.

PERVASIVEHEALTH 2010, March 22-25, Munchen, Germany

Copyright @ 2010 ICST 978-963-9799-89-9

DOI 10.4108/ICST.PERVASIVEHEALTH2010.8911 
market, influencing its future development [4]. Even if this cycle cannot be broken, the new trend on adaptable interfaces can help seniors overcome this lack, through allowing them to adapt ICT tools to their needs.

The above tendency is no more evident in social networks which are the popular phenomena amongst younger generations, who connect with them in vast numbers, and are designed and built around applications with this sector of the society very much in mind. Facebook, Bebo and Youtube for instance have made ICT a very important force in mobilizing the social capital of a younger generation, who both relate to this field of communication and are savvy enough to adapt it to their own user generated content. Societal networks and interconnections are continuously expanded and enriched by new content. But since this environment is very much designed with the younger user in mind, seniors are wary of and feel excluded from its use. Furthermore, these networks provide a level of adaptability which is again aimed largely at a younger generation and while successful, do not help developing online cultures among seniors.

Through ePAL's technological perspective, three preconditions are specified as required for the success of extending professional active life of seniors, including:

- Availability and use of computing resources e.g. pervasive computing and communication links for all senior professionals must be supported.

- Establishment of virtual communities of seniors to work together on solving emerged problems in the market/society, and performing individual and/or joint tasks.

- Provision of advanced ICT technology and support tools targeting senior professionals and their working/co-working.

The roadmapping strategy of the ePAL has identified four fundamental ICT related areas in need of further research and development for support of the specific requirements and characteristics of senior professionals and their continued enjoyable active working and co-working, namely:

- Collaborative Networks. Specification of a reference model for ePAL environment to capture all its endogenous elements (i.e. components, structure, functions, and behavior governance), as well as the exogenous interactions (with the constituents, market, society, and support providers). This model is required to facilitate the study, understanding and developments for ePAL.

- Affective Computing. Design and prototyping tools and systems to support the needed functionality and operation, with specific attention on behavioral aspects of senior professionals and ePAL communities.

- Soft Computing. Design new approaches and tools to on one hand reduce risks and frictions, and on the other hand boost and motivate collaboration and co-working among ePAL stakeholders. Here the issue of trust, mediation, rewards and incentives, value systems, etc. comes up which all deal with incomplete and imprecise information, and thus their soft modeling and qualitative analysis are required.

- Machine Learning. Specification of models and tools assisting seniors to leave their legacy behind, learning from their practices. Designing new tools for decision making through learning from past experiences and through ranking solutions with pre-defined indicators.

This paper mainly focuses on the survey of promising technologies for ePAL communities, which in one way or another can be used to smoothen and facilitate the involvement of retired professionals in daily society activities. It also addresses a number of challenges in need of future work on these promising technologies in support of effective operation of communities of retired professionals.

There are a number of new ICT technologies and advances which show to certain level, the potential to facilitate the extension of professional active life amongst seniors. However, at present on one hand, sustainability of existing freeware technologies is not clear and on the other hand there is a real need for their advancement to be steered towards the needs of senior professionals.

In order for advances in ICT systems to have the desired effect they should be developed with a proper understanding of the needs of those who will be using them. This may seem an obvious point, but it would appear important that the technological aspects of ePAL advance in parallel with its societal and organisational models. In a highly competitive industry which develops at a rapid pace applying a constantly updated line of ICT services in which new advances quickly become redundant, would mean too much of a barrier to its normal development. One solution to this problem, which is very much in line with present technological trends, is to offer a high degree of built-in adaptability to new technology. Advances in "configure yourself" technology and support systems for user generated knowledge content, along with easily customizable interfaces are crucial to allow brokers and senior professionals themselves to react to changes in a non static marketplace. Where seniors are working with younger professionals' adaptable technology would have to be compatible and not virtually alienate senior professionals from their co-workers.

The introduction of new working patterns, relying for instance on remote collaboration of teams of senior professionals who interact with brokerage entities and customers, also introduces the need for provision of new infrastructure functionalities and supporting tools. The subsequent sections further addresses in details the main identified concepts related to the technological perspective, namely: collaborative networks, affective computing, soft computing, and machine learning.

\section{COllaborative NETWORKS}

As a guiding work hypothesis, ePAL advocates the development of collaborative networks involving senior professionals and other supporting actors of the socioeconomic system. In line with other initiatives in related areas, e.g. eSangathan, the ePAL initiative assumes that an effective transformation of the current situation regarding retirement, and the barriers to active ageing in Europe requires the introduction of new approaches and ways to create actively contributing professional communities in society. These communities will support the elderly citizens with a framework for leveraging their talents and expertise and creating value for the benefit of the Europe's economy. Moreover, the framework would also support a balanced transition towards stepwise retirement. Taking advantage of current advances in ICT technologies and in line with current 
trends in several European regions, the success of extending professional active life of seniors (ePAL) requires establishing $2^{\text {nd }}$ generation professional virtual communities support senior professionals with remaining active and involved [1] in the market and society. This section presents the base concepts of collaborative networks and their applications in communities of active senior professionals.

Base concepts of collaborative networks. A traditional Virtual Community is composed of a group of individuals that virtually come together, i.e. by means of computer communication, and stay in touch for a long period of time [5]. Through Virtual Community, the members interact with each other creating personal relationships, sharing common social ties in the form of interests, feelings, knowledge, and experiences [6]. The aim in ePAL is to step beyond "socialization", and to pursue mechanisms for interaction with the socio-economic system, and therefore facilitate the use of the potential contributions from retiring and senior professionals.

A Collaborative Network $(\mathrm{CN})$ is a network consisting of a variety of entities (e.g. organizations, people, even intelligent machines) that are largely autonomous, geographically distributed, and heterogeneous in terms of their operating environment, culture, social capital and goals, but which decide to collaborate to better achieve common or compatible goals, and whose interactions are supported by computer network [7].

Among the CNs, we can distinguish between the long-term "strategic" alliances and the goal-oriented networks. Longterm strategic networks/alliances are established to act as the breeding environments for goal oriented networks, namely with the purpose of getting their participants prepared for participation in response to collaboration opportunities. In other words, they are alliances aimed at offering the conditions and environment to support the rapid and fluid configuration of goal oriented collaboration networks, when opportunities arise. Goal-oriented networks are CNs in which intense collaboration (either towards a common goal or a set of compatible goals) is practiced among their partners, for example the virtual organizations (VOs). All CNs aim at value creation, while the value system of each $\mathrm{CN}$ is defined to serve the specific short or long term aims of that $\mathrm{CN}$.

In this paper we address two specific forms of CNs, namely one short-term type (i.e. VOs) and one long-term type (i.e. Virtual organizations Breeding Environments -VBEs). VOs represent short-term goal-oriented collaborations between partners, while VBEs represent long-term cooperation. Although their main actors involve organizations, the base concepts have the potential to be adopted, tuned and applied in network settings involving individuals such as senior professionals.

Organizations interoperate and collaborate within VO and VBE networks while being facilitated by computer networks, in order to achieve certain common or compatible goals, such as the acquisition of and response to larger, better, and more business opportunities. As such, different kinds of co-working are applied in short-term and long-term CNs and in order to further describe and distinguish between the cooperation and collaboration concepts related to $\mathrm{CNs}$, the following definitions are applied in this paper [8]:

Cooperation, practiced in long-term CNs, involves not only the exchange of information and alignment of activities, but also the sharing of resources for achieving compatible goals. Cooperation is achieved by the division of some minor labor (not extensive) between participants. However, a common plan exists that in most cases is not defined jointly, but is designed by a single entity (perhaps by the coordinator/administrator of the cooperation alliance), and which requires some low-level of cooperation.

Collaboration that is practiced in short-term CNs on the other hand is a process in which entities share information, resources and responsibilities in order to jointly plan, implement, and evaluate a series of activities that will help them achieve the common collaboration goal. It implies a group of entities that work intensively together and enhance each other's capabilities. It also implies sharing risks and rewards that, if desired by the group, can also provide outside observers with the impression of a joint identity. Collaboration involves the mutual engagement of participants to solve a problem together, which requires strong trust relationships and thus takes time, effort, and dedication.

As a basic rule, supporting the dynamic/fluent formation of collaborative networks, such as in a shorter term consortium, requires its potential partners to be ready and prepared to jointly participate in such a collaboration environment. The foundation of this readiness should include reaching commonality agreements on aspects such as the interoperable infrastructure, operating rules, and cooperation. Any collaboration also requires that all involved actors meet the required level of competency and performance to be considered trustworthy by other partners. Therefore, the concepts of long-term strategic alliances have emerged as the necessary context for the effective creation of dynamic short term consortiums.

A main aim of the long-term strategic alliances is focused on the transition from point-to-point connections between actors to a network structure in order to increase the chances of its members' involvement in opportunities for collaboration, and to reduce the costs and time needed to configure opportunity-oriented consortiums.

Strong relevance of collaborative networks' concepts to the ePAL communities. ePAL communities can also be established on the basis of concepts related to collaborative networks. While characterizing the collaboration among senior professionals we have introduced the following terms related to collaborative networks of senior professionals to support characterization of the ePAL communities [1].

CASP (Community of Active Senior Professional) constitutes an association of senior professional individuals that are largely autonomous, geographically distributed, and potentially heterogeneous in terms of their: capabilities, offered capacities, culture, system of values, etc., but sharing their main compatible and/or common goals of increasing their active professional life in the society and/or market, through co-working with others in Teams of Senior Professionals supported by computer networks, 
under the slogan of: "Together Everyone Achieves More!".

TSP (Team of Senior Professionals) is a collaborative network of individuals configured and established within the CASP in response to opportunities in the society and market that are in need of their wisdom and/or knowledge assets that they can offer, which as a consequence supports the retired professionals remaining professionally active.

$\boldsymbol{S P}$ (Senior Professional) is a retired professional who becomes a member of CASP in order to increase his/her chances of staying active and involved in the socio-economic system, and perhaps also through getting involved in potential TSPs established through the CASP.

Individuals decide to join and collaborate in professional communities when they are convinced that they can achieve more by co-working with others. Such achievements may include: optimizing their business profits, improving their knowledge and skills, complementing themselves with missing resources and competencies. The advantages of the geographical and societal widening are in this respect evident: the widest the social and geographical spread, the highest the possibilities for the individuals to reach their objectives.

An association of retired professionals can represent a special form of a professional virtual community. The establishment and management of such alliances of individuals can benefit from well researched and developed concepts and tools for professional virtual communities. Most collaborative networks are business oriented entities, particularly focusing on supporting their members to jointly optimize individual financial gains. Therefore, the focus of associations of retired professional is not necessarily enhancing the financial gains of members. Rather to keep and enhance the professional active life of their members. With support of CASPs senior professionals can remain professionally active and thus involved in the society. These seniors are willing to transfer, share and exchange their knowledge and experience to those individuals or organizations that are in need. There are two challenges that retired professionals are facing when providing their services in terms of bundles of knowledge and experience to beneficiaries, as described below:

o Opportunity brokerage: Finding opportunities to apply their knowledge and experience - i.e. beneficiaries willing to accept their offers - is quite challenging. From our empirical study it was observed that many retirees within existing CASPs face difficulties when trying to individually acquire opportunities in the society such as consultancy, since those are also being targeted by other formal service providers. Even when such opportunities are brokered for senior professionals, the process of building trust and credibility for the individual retirees in the eyes of the beneficiaries might be very challenging.

o Opportunity complexity: Most acquired opportunities cannot be responded by one senior professional alone. For example, SMEs which need consultancy on a subject typical require more than one kind of expertise need a number of seniors to collaborate, in order to provide the required services

Further required research on collaborative networks applicable to ePAL environments. Solutions on challenges related to future collaboration, to enhance a fully realization of promises of electronic co-working, require advancements in networking technologies, tools and systems, user interfaces and interaction paradigms. It also demands, advanced interoperation between applicationspecific components and generic supporting tools. Below we present some topics in need of further research in relation to application of concepts related to collaborative networks namely CASPS and TSPs to ePAL environment.

\section{(i) Specification of formal conceptual models for people's professional life cycle and the support environment for active ageing}

In research, there is still lack of suitable formal conceptual models to support regulating people's professional active lifecycle. It is essential to establish a reference model for the extension of professional active life and active ageing, The model should incorporate the elaboration of a common ontology for the senior professional communities. Another component that must be supported is the development of contractual and business models for CASPs. As such, in relation to development of formal conceptual models, the following need to be further addressed: (i) Development of conceptual models to support designing and implementing of collaboration support services, including services supporting (virtual) teams' formation and management that are user friendly to senior professionals; (ii) Discovery and management of collaboration ontology that supports a variety of stakeholders in CASPs and configured teams of senior professionals; (iii) Design of templates for novel contractual and cooperation agreements, as well as designing models to support the implementation systems supporting negotiation among senior professionals; (iv) Development of models to guide the implementation of advanced marketing and brokerage services; (v) Designing new networking models for elderly communities' involvement with the socio-economic system.

\section{(ii)Development and integration of self-adaptive and configurable technology solutions in ICT environments, to enable customized involvement of seniors}

Self adaptive and configurable technology is able to evaluate its own behavior and change its behavior to improve itself based on its present evaluation result [9]. For example, it can change its behavior to improve its performance when evaluations result indicates that it is not accomplishing what the technology is intended to do, or when better functionality or performance is possible [10]. Thus the technology also modifies its behavior in response to changes in its operating environment - e.g. end user input, availability of external hardware device, etc. This implies that the self-adaptive technology, such as a computer system, has multiple ways of accomplishing its purpose, and has enough knowledge of its construction to make effective changes during runtime. Such technology should include functionality for evaluating its behavior and performance, as well as the ability to re-plan and reconfigure its operations in order to improve its operation. Self adaptive technologies should also include a set of components for each major function, along with descriptions of those components, so that components of the systems can be selected and scheduled at runtime, in response to the 
evaluations. It also requires the ability to match input/output of sequenced components, and the ability to generate some of this code from the specifications.

There are some seniors that may require special training and assistance when dealing with technology. It is thus desirable and/or necessary to have self-customizable tools supporting collaboration operations that empower seniors to better use the provided ICT solutions. For this challenge a number of technical solutions may be developed, such as: selfadaptive interface systems and technology assistance wizards. To deal with emerging technologies, seniors should also have access to several training modules. In relation to this topic further research is required to address the development of "Configure yourself" -based infrastructure, and easily adaptable and customizable user interfaces. Relevant and challenging aspects in this area that are in need for further research include the following topics: (i) Identification of generic and customizable indicators for measuring the performance of systems/tools during runtime; (ii) Design and developmentment of mechanisms supporting systems to make decision regarding changing their behavior based on collected performance data; (iii) Development of affective/emotional tools to detect and discover state/status of interactions between systems and senior professionals (addressed also later).

\section{(iii) Development of ICT collaboration platforms, enhanced by affective computing, context awareness, and trust establishment, to support operations of CASPs}

ICT collaboration platform offers a set of software components and software services that enable actors to find each other and the information they need to be able to communicate and work together to achieve common business goals. Its core elements typically include: email, calendaring, scheduling and contacts, file synchronization, wiki of ideas and notes, task management, full-text search, instant messaging, web conferencing, application / desktop sharing, voice, audio and video conferencing, blog, wiki, tagging, RSS, shared bookmarks, etc. as initiatives such as eSanghatan project [2] have also investigated.

In the context of CASP it is of extreme importance to be supported with an ICT collaboration platform for communities of senior professionals. Such a platform, besides providing the basic required services (as addressed above) constitutes part of the governance systems of this community. Thus, it should also provide operational services/tools such as an advanced management system for CASP, a trust building management system, together with users/community affections / emotions management system. To realize a proper ICT collaboration platform for senior professionals, further research need to be performed addressing the following: (i) Development of advanced collaboration support services, including (virtual) teams' formation and management, (ii) Development of affective computing and context aware enriched environments, (iii) Formulation of mechanisms and development of systems supporting the establishment of trust among stakeholders, (iv) Provision of security and ethical / privacy support.

(iv)Design and development of support collaborative tools

\section{and systems to facilitate value creation in CASPS} environment

For ePAL, effective marketing is essential to the success of business brokerage. Communities of senior professionals should be supported to assess their business climate and capabilities in order to enhance their identity of brokerage brand through marketing strategies. Such strategies may include advertising, public relations, customer service efforts, etc. Considering the current advancement of technologies, supporting collaboration among actors, marketing related processes require to be properly supported by collaborative tools and systems to enhance their effectiveness. Therefore, in order for collaboration platform to enable its community members in performing their professional activities, it must provide facilities and tools for the following processes: (1) Marketing and brokerage, (2) Virtual Team creation, (3) negotiation and e-contracting, (4) Virtual Team management, (5) collaborative problem solving support, and (6) Intellectual Property and performance management, etc.

Furthermore research needs to be performed to address the development of the tools and systems supporting the following: (i) Advanced collaboration support services, including (virtual) teams' formation and management, (ii) Tools supporting the process of value creation, (iii) Advanced marketing and brokerage services, (iv) Security and ethical / privacy support.

\section{AFFECTIVE COMPUTING}

The establishment of CASPs is based on the premise that 'professional society' and senior professionals can mutually benefit from actively re-involving retirees in the economic process. Technology might be used to bridge the gap between retired professionals and organizations by providing facilities to create contact, collaborate and recognise needs on both ends. However, only focusing on the provision of functionality to facilitate cooperation is not enough. Rather, motivating all parties and ensuring a positive experience will be just as crucial for professional interactions among these age groups.

Retirees are not obligated to work, nor are organizations obliged to include retirees in their daily work practice. For this to be realized both retirees and organizations need to perceive value in continuing participation. Any technology used to facilitate active ageing will have to be designed taking into account a selection of social and affective aspects of interacting with computers and each other online [11]. Examples of such affective aspects include: (i) Targeting and managing (online) individual identities, (ii) Adapting to the specific needs of the individuals on both ends, (iii) Matching organizations and professionals, (iv) Overcoming challenges of online communication, (v) Fostering feelings of inclusion and value, (vi) Establishing trust in the technology used, trust in each-other and trust in one's own ability to contribute.

Affective computing deals with emotions. Emotions themselves are very human matter, of which there is no clear theory or understanding related to how they are created or how they evolve. However, affective computing could provide massive improvements in human-computer interaction, in the 
form of usable and intuitive interfaces [6]. Affective computing deals with the design of systems and devices that can recognize, interpret, and process human emotions [12]. It is an interdisciplinary field spanning computer sciences, psychology, and cognitive science. While the origins of the field may be traced as far back as to early philosophical enquiries into emotion, the more modern branch of computer science originated with Rosalind Picard's 1995 paper on affective computing [13]. A motivation for the research is the ability to simulate empathy. The machine should interpret the emotional state of humans and adapt its behavior to them, giving an appropriate response for those emotions.

The latest scientific findings indicate that emotions play an essential role in decision making, perception, learning and more. They influence the very mechanisms of rational thinking. Not only too much, but also too little emotion can impair decision making. According to Rosalin Picard [13], if we want computers to be genuinely intelligent and to interact naturally with us, we must give computers the ability to recognize, understand, and even to have and express emotions.

Further required research on affective computing applicable to ePAL environment. In short, taking into account the characteristics of the involved individuals, their societal context and (affective) experiences involved in the mutual cooperation - without relying on pre-existing assumptions about these concerns - could prove key in the success of collaborative technologies that facilitate active ageing

(i) Development of approaches that discover patterns and model 'the evolution of senior professionals' interests and their involvement in the socio-economic system" and "the behavior and emotional health of senior professional networks".

Behaviors of people at certain point in time are influenced by characteristics of the environment in which they are operating, which in turn influence the people's interests and decision on performing certain activities. Specifically for communities of active senior professionals, further research is needed to enhance the development of a conceptual base for behavioral modeling, data-mining / machine learning approaches for behavioral patterns discovery, forecasts and simulation methods and tools for behavioral analysis. Furthermore, models and tools need to be designed and developed for supporting management of emotional health of collaborative networks. In relation to this topic further research should also address the following among others: (i) Develop approaches to characterize emotions and define necessary differentiating indicators, (ii) Development of easily adaptable and customizable user interfaces that are emotional supportive, (iii) Development of tools supporting affective computing and that are context aware enriched environments, (iv) Formulation of mechanisms and development of systems supporting the establishment of trust among stakeholders, (v) Design of new networking models for elderly communities' involvement with the socio-economic system.

(ii) Development of approaches and tools providing support for sensing and recognition of emotions of SPs

The range of means and modalities of emotion expression is so broad, with many of these modalities being inaccessible and many others being too non-differentiated. This makes it difficult to collect the necessary data to perform the required affective related computation. In fact, people's expression of emotion is so idiosyncratic and variable, that it is challenging to accurately recognize an individual's emotional state from the available data. This subject still needs further research and development in both aspects of affective computing technologies, such as automated systems, and hardware, such as sensors.

(iii) Design and development of modelling approaches and tools for seniors' emotions and their related evolutions

There is still a lack of suitable models developed to support the design of required affective computing related systems, and not enough progress has been made with cognitive modeling. While some data exist regarding the effects of emotion on decision making and behavior, the majority of these data were collected in highly artificial environments, such as laboratories, and both their robustness and application seem to fit the need of academic and research needs but not for empirical need in real life. Existing models of emotion use highly stylized stereotypes of personality types and emotional responsiveness, which do not correspond to real behavior in people. Together, these factors make it difficult for any existing single modeling approach and/or tool to provide valid models of emotional processing.

(iv)Design and development of tools and systems that can process and simulate the evolutions of emotions of senior professionals to support prediction of possible future behaviour

Upon collecting data regarding emotions of senior professional an extensive analysis is needed to understand their evolution with time. The examination of this data should include simulating different scenarios to predict possible future behaviour and emotions of senior professionals. With proper simulation results and well defined scenarios an analysis can be done to identify indicators that can be used to show the cause of changes in emotional and behaviour of senior professionals.

(v) Development of interfaces for displaying, communicating, mediating and expressing SPs' emotion

Technology that sends affective information - portraying affect through some modality - might be possible to build. However, the hardest challenge in real-time interaction is figuring out when to communicate which affect. Without understanding social display rules and other important cues about the interaction context, technology is quite likely to irritate people with its emotional outputs.

Furthermore, the difficulty of communicating emotions between two nodes through text-based online interaction has led to the development of emoticons and other means for people to add affective intent. The extents to which these attempts are based on well formulated principles and actually work to properly communicate emotion are topics in need for 
further research.

Moreover, some people prefer non-affective interaction with computers and do not wish for computers to communicate social-emotional signals. Affective computing designers can honor this preference by designing such aspects of control or adaptation. Since a driving principle in the development of affective computing is to honor people's affective preferences in the technology design, the preference of "show me no affective communication" should be accommodated. How will systems automatically reconfigure themselves to react to such situations is a topic also in need for further research.

\section{(vi)Establishment of affective computing related ethics for} SPs

In real life, emotions, perhaps more so than thoughts, are ultimately personal and private and thus people are not willing to see their emotions documented especially when they visualize those people negatively [14]. Any attempts to detect, recognize, not to mention manipulate, a user's emotions thus constitutes the ultimate breach of ethics that will be difficult to be accepted by computer users. Thus in relation to ethics, further research needs to be done to find ways on how ethical rules can be tuned to facilitate the adoption of affective computing in daily life activities.

\section{(vii)Intelligent and respectful system responses to} perceived emotions for SPS

When a person reveals affective information to a recipient, the recipient can choose ways to respond that may be helpful or harmful. For example, if a person lets a computer (robot or agent) know that its action is very frustrating then the computer could try to recognize its gaffe and take steps to avoid it in the future. It could issue an acknowledgement of the frustration it has caused, and perhaps even apologize, and see if this helps alleviate the person's frustration. Sometimes it might be appropriate for a computer to display an empathetic or caring response. Computer-provided empathy can reduce frustration and stress and can impact perceptions of caring, which could help in health-care technologies, among others. The challenge in relation to these issues is on how they will be addressed and incorporated in systems used for affective computing.

(viii) Design and development of affective qualified collaborative systems that are user friendly to senior professionals

Every senior professional can achieve more and better when participating in collaborative teams that working alone. The introduced concepts of CASPs and TSP in this paper demonstrate the need for well configured and supported collaboration among senior professionals. For this purpose collaborative systems need to be designed and developed taking into consideration the usability and provided functionalities that are suitable to senior professionals.

\section{SOFT COMPUTING}

The development of CASPs with an active involvement in the socioeconomic system represent a complex case whose proper understanding, design, implementation, and management require the integration of different modeling perspectives and techniques. The advances in soft computing may lead to the creation of more flexible software that can adequately help researchers to deal with modeling complex systems that are related to ePAL communities. As a specific form of collaborative networks, CASPs are also characterized by their increasing levels of uncertainty, complexity, and heterogeneity, which are the complexities now addressed under soft computing.

Soft computing differs from conventional (hard) computing in that unlike hard computing, it is tolerant of imprecision, uncertainty, partial truth, and approximation. In effect, the role model for soft computing is the human mind [15]. The guiding principle of soft computing is: exploit the tolerance for imprecision, uncertainty, partial truth, and approximation to achieve tractability, robustness and low solution cost [16].

Perspectives related to behavior and decision making in face of incomplete and imprecise information are particularly difficult to model [15]. In fact, this context involves a number of autonomous and heterogeneous entities - organizations and people - often with: (1) different value systems, and therefore different perceptions of value and importance / priority of things, and (2) a behavior influenced by factors such as emotions, health / physical status, preferences, working habits, ethical values, level of trust, competences, etc.

Nevertheless, and in spite of these difficulties, entities involved in a collaborative network need to plan and make decisions in scenario cases with incomplete and imprecise information [15]. This raises the need for: (1) Modeling approaches and developing models to represent such contexts; and (2) Reasoning techniques for decision-making in contexts of incomplete and imprecise information.

Other dimensions of this problem area include "complexity", what researchers try to capture with the notions of non-linear models, emergence, self-organization, etc, as well as the "dynamism" of the networks in terms of their "shape / topology" (membership, roles, distribution in case of mobility). Soft modeling methods can exploit the tolerance for imprecision, uncertainty, and partial truth, and are therefore promising candidates to deal with the issues addressed above.

One characteristic of soft computing is the intrinsic capability to create hybrid systems, combining two or more soft computing (SC) methodologies to benefit from consequent synergistic effects [15]. Such arrangement provides complementary reasoning and searching methods that allow combining domain knowledge and empirical data (e.g. training data sets) to develop flexible computing tools and solve complex problems. Figure 3 illustrates a taxonomy of these hybrid algorithms and their components.

Some typical application areas of soft computing are data mining, pattern recognition and classification, optimization, decision support systems, and control systems. A study of applicability in collaborative networks, including an extensive set of case studies can be found in [8]. Soft computing includes both the "modeling" of imprecision, partial truth, and 
uncertainty - also referred to as soft modeling - and the "reasoning" and inference based on such models - also referred to as soft reasoning. Therefore, soft computing refers to soft modeling plus soft reasoning.

In addition to the classical methods of soft computing as the ones mentioned above, there are other approaches with potential applicability in dealing with vagueness. One example is qualitative reasoning, which creates simplified representations for continuous aspects of the world, such as space, time, and quantity, which support reasoning with very little information [17].

Below we discuss few application examples of soft computing in communities of active senior professionals.

\section{(i) Quantitative analysis of factors in active ageing environments:}

In the discipline of system dynamics [18] it is assumed that a complex system can be broken into smaller measurable elements (criteria). The criteria can then be analyzed using the causal modeling approach to study their causal behavior. The results of causal analysis can be represented in a diagram showing the relations among the criteria. These causal relations can also be translated into mathematical equations. The mathematical equations can be applied to make a formal analysis of the entire system. Figure 4 shows the application of the causal modeling approach to the system dynamics discipline; namely, first the generation of System dynamics based on causal models, followed by the definition of mathematical equations that lead the way to the design of the system architecture [19]. In principle, these concepts could be applied to analyze different measurable factors that causally influence the activeness of retired people. The analysis results, once translated into mathematical equations, can be a base for making formal analysis of factors that causally influence the elder's activeness. The translated equations can also support the automation of some processes related to the analysis of factors influencing elder activeness.

One practical example application of quantitative causal reasoning could be the analysis of the impact of changing the taxation rules for activities involving retired people.

\section{(ii) Qualitative analysis of factors in ePAL environments}

In the discipline of qualitative reasoning (QR) it is assumed that some complex systems are characterized with parameters which are difficult to quantify or their measurable data are hard to collect. However, such systems can be analyzed through developing their QR based models that can be used to identify some values for these parameters with some qualitative degrees (e.g. large, medium, small, etc.). Furthermore, based on these qualitative values, the models can be simulated to analyze the causal influences among these qualitative parameters which represent the high level behavior of the entire system. The results of the causal analysis can then be applied to qualitatively examine the entire system [19].

Qualitative analysis has been popularly applied in study of populations of living things. It has been for example applied to study population changes of particular species of plants in ecological studies. The ecological knowledge gained from a particular study is usually characterized as incomplete, fuzzy, uncertain, sparse, empirical, non-formalized, etc., and it is often expressed in qualitative terms, verbally or diagrammatically [17]. Qualitative reasoning is an area of artificial intelligence that creates representations for continuous aspects of the world to support reasoning with little information. Particularly relevant for the study addressing imprecise situations is the support for analysis of qualitative parameters addressing their explicit representation of causal relationships between values of parameters. There are a number of aspects regarding activeness of retired professionals that can hardly be quantitatively characterized and measured. In the ePAL context, for instance, decisions made by retired professionals regarding joining some economical activities are causally influenced by many factors (e.g. possible benefits, motivation, impact to their health, impact to their social contacts, etc.) that cannot easily be studied with traditional mathematical approaches.

\section{Further required research on soft computing - Applicable to ePAL environments}

A key challenge related to soft computing research lies in judicious integration of the merits of its component technologies so that the resulting one has application-specific advantages, which cannot be achieved using the individual techniques alone, in decision making. Although the soft computing as well as qualitative reasoning fields has addressed diverse problem areas and developed a variety of theories and systems, there are a number of prominent features that are typical for many of the approaches. Some of the most important ones are as follows:

\section{(i) Development of ontologies for various concepts in CASPS collaborative environments}

There is a need for providing an explicit representation of the conceptual modeling layer for example as addressed in studies in the qualitative reasoning field. This layer is crucial to any attempt to support model building and even more to automate it and has been one of the major issues of the field. Particularly, for its application in collaborative environments further research needs to be done to enrich ontology specification which will support studies related to soft computing.

\section{(ii) Development of approaches to support or enhance analysis of causality for CASP related variables}

Analyzing and explaining the behavior of a system and its constituent variables in terms of cause-effect relations is central to human intuitive reasoning and communication but it is challenging to formally formulate mathematical expressions describing system behavior. Formalizing this concept and exploiting it in automated reasoning is the basis for explanation facilities in model-based systems which is still in need for further research.

(iii) Development of an approach and a framework for compositional modeling of CASP elements

Most qualitative reasoning systems adopt a reductionist 
view of the world and aim at building libraries of elementary, independent model fragments (component behavior, processes, and so on). This approach provides the basis for automating the model composition and reusing models, a highly desirable feature for industrial applications.

\section{(iv)Addressing the qualitativeness of systems supporting operations in CASPs}

Qualitativeness means including only those distinctions in a behavior model that are essential for solving a particular task for a certain system. The goal is to obtain a finite representation that leads to coarse, intuitive representations of models and behavior (for example, as finite relations and a state graph, respectively), and efficient algorithms.

\section{MACHINE LEARNING}

For the ePAL context, examples of potential use of machine learning and data mining techniques include:

- Discovery of behavioral patterns of elders in communities provided historic data is collected regarding the participation of members in the activities of the community.

- Identifying the "emotional health" status of a collaborative network of senior professionals by monitoring some selected indicators (in relation to affective computing).

- Optimization of collaboration processes based on historic data on past performance.

As mentioned above, many machine learning techniques are already available being the main challenges here the integration with the other components of a virtual community support system and the collection of adequate (historic) training data sets. Machine Learning is the science of building hardware or software that can achieve tasks by learning from examples [20]. The examples often come as \{input, output pairs. Given new inputs a trained machine can make predictions of the unknown output. Examples of machine learning tasks include: automatic reading of handwriting, assisted medical diagnosis, automatic text classification (classification of web pages; spam filtering), and stock exchange predictions.

Further required research on machine learning - applicable to ePAL environments

Further research on different topics related to machine learning must be done to facilitate the designing and development of algorithms that allow the related concepts to be applicable in networks involving individuals such communities of active senior professionals. Below we present a few representative topics that are in need for further research.

(i) Develop machine learning-oriented tools to support the establishment of environments that empower seniors to leave a legacy that capitalizes on their personal / professional experience and is usable and transferable to the society

Personal and professional experience of seniors' should be made available to be used by other society generations, namely the younger generations, or to allow the other members of the senior community to benefit from it. This legacy can be used if mechanisms to promote intergenerational inheritance and collective legacy are developed. These mechanisms should use other instruments such as a talent modeling and user-centered knowledge acquisition tools. These mechanisms should consider the importance of the models for knowledge assessment and intellectual property. Further research is needed to address the following: (i) Development of tools supporting inheritance and sharing of user-generated knowledge assets, (ii) Design of new networking models for elderly communities' involvement with the socio-economic system, (iii) Develop tools that support learning achievement of senior professionals to facilitate the rewarding process.

\section{(ii) Addressing the computational limits of machine learning} for large volume data in CASPS

Communities of active senior professionals are open but controlled border networks and thus they might be characterized with extremely large datasets which are constantly growing. Therefore, a related research challenge in need for further study is finding solution related to building effective learning algorithms that are operative in CASPs with very low computational cost required for test time.

\section{(iii) Development of reduction methods addressing label complexity for data related to SPs}

Considering the complexity of the CASPs as collaborative networks, it is very challenging to collect the data related to senior professionals that is needed to provide their required experience for machine learning purposes. The data in collaborative environment is very dynamic and can be in a very large variety and volume. The classification and labeling of the variables representing the data is quite challenging. In many situations there are also sometimes very small amounts of labeled data, because some labels are costly, or because the prediction task to solve is very specific. In relation to labeling, two open questions that need further research refers to (1) What are the limits of multitask learning and active learning? And (2) How to reuse knowledge bases \& ontologies representing concepts of senior professionals and CASPs in machine learning?

\section{(iv)Addressing the problem of analyzing non-stationary data related to $S P$ s}

The standard assumption in machine learning is that data sources do not change, but the data and their related sources in communities of active senior professionals are very dynamic. Thus some learning algorithms and tools need to be tuned to deal with changing data and data sources in CASPs. This is an open challenge in need for further research.

\section{(v) Addressing the difficulties in learning to rank various solution for emerging opportunities in CASPs}

There exists a large set of solutions from which an optimal one will need to be selected, for instance for decision-making in CASPS in relation to formation of TSPs. As a step towards the needed selection, it requires establishing ranking mechanisms for all solutions based on certain set of indicators. A key challenge in need of further research is related to 
establishing the ranking policies and identifying the suitable indicators for various types of solutions.

\section{CONCLUSION}

Although there are still a large number of challenges related to various research disciplines that need to be addressed to smoothen the establishment and operation of networks of senior professionals, those related to technologies and applications are amenably more in number and strongly in need for extensive innovation. This paper has presented a number of representative topics related to the technological perspective of ePAL communities. For each presented topic namely, those of collaborative network, affective computing, soft computing, and machine learning, a number of specific challenges that are in need for further research are introduced and discussed.

From previous and on going research projects some work in relation to development of tools required for the general $\mathrm{CN}$ areas addressed in this paper have already started and a few have made substantial progress [21]. This indicates that in order to fully achieve the desired vision of the ePAL [1], both the complexity-level as well as the required R\&D vary among different challenges presented in this paper. Below are a few examples addressing this issue in more details:

- During the last years a number of research projects have addressed different aspects of collaborative networks. A large volume of innovative solutions are addressed in proceedings of the PRO-VE conferences 1999-2009. Thus solving some challenges presented in this paper in the area of collaborative networks might require customization or application of some existing solutions to the associations of senior professionals, while some others such as challenge (ii) I Section II, requires R\&D on requirement analysis and development of corresponding support functionalities.

- The mentioned area of affective computing on the other hand is a new area of research and development, and has many unresolved challenges related to capturing and processing emotions of senior professionals in their collaborating associations.

- Developing many CN-related support tools rooted in the areas of soft computing and machine learning is also a new area in need of formal in-depth specification, modeling and development of innovative tools, e.g. challenges (ii) and (iii) in Section IV, and challenges (i) and (v) in Section V of the paper.

Therefore, the presented research challenges aim at identification of needed enhancements to the current research and development achievements in the four related areas, in order to meet the demands and requirements for realization of the technological perspective of the ePAL environment; thus for establishing communities of active senior professionals.

\section{ACKNOWLEDGMENT}

This work was funded in part be the European Commission through the ePAL project.

\section{REFERENCES}

1. Afsarmanesh, H., Camarinha-Matos, L.M., \& Msanjila, S., A wellconceived vision for extending professional life of seniors. In proceedings of PRO-VE 2009 conference, Springer.

2. Turbe-Suetens, N., Kouloumdjian, M.-F. Active Ageing Workforce in Virtual Environment - Methodology and Innovation - eSangathan White Paper, http://www.scribd.com/doc/6121465/Active-Ageing-Workforce-inVirtual-Environment-Methodology-and-Innovation-eSangathan-White-Paper

3. Hernandez-Encuentra, E. Pousada, M.\& Gomez-Zuniga B. ICT and Older People: Beyond Usability. In Educational Gerontology, Special issue on Adult Education and Lifelong Learning. Volume 35, Issue 3, pp $226-245$, (2009).

4. Lundberg, S. Elite care brings elder friendly technology to Oregon. In the bimonthly newspaper of the American society on ageing - special issue on ageing today. Vol xxviii, No. 5, ISBN 1043 1284, September 2007.

5. Crave, S., Bouron, T. \& Ladame, S. Using social capital as a conceptual framework for professional virtual communities formalization. In IFIP International Federation for Information Processing, vol. 224, NetworkCentric collaboration and supporting framework, pp. 317-378. (2006)

6. Picard, W. Adaptive collaboration professional virtual communities via negotiation of social protocols. In IFIP International Federation for Information Processing, vol. 224, Network-Centric collaboration and supporting framework, pp. 353-360. (2006)

7. Camarinha-Matos, L.M. \& Afsarmanesh, H. Collaborative networks: Value creation in a knowledge society, (invited keynote paper) in Proceedings of PROLAMAT 2006, IFIP Int. Conf. On Knowledge Enterprise - New Challenges, Springer, Shanghai, China, (2006).

8. Camarinha-Matos, L.M. \& Afsarmanesh, H. Classes of Collaborative Networks. In the Encyclopedia of Networked and Virtual Organizations (G. Putnik, M. M. Cunha, Eds.), Idea Group. January 2008.

9. Laddaga, R., Robertson, P. \& Shrobe, H. Introduction to Self-adaptive Software: Applications. In Self-Adaptive Software: Applications, Lecture Notes in Computer Science, ISBN 978-3-540-00731-9, LNCS 2614, pg. 275283, (2003).

10. Osterweil, L. J. \& Clarke, L. A. Continuous self evaluation for the selfimprovement of software. In self adaptive software, ISBN 3-540-41655-2 pg 27-39, (2001).

11. Cramer, H.S.M. Interaction with user-adaptive information filters. Trust, transparency and acceptance. CHI ' 07 extended abstracts, San Jose, Ca, USA, pp. 1633 - 1636, (2007).

12. Lee, C.M., Narayanan, S. \& Pieraccini, R. Recognition of Negative Emotion in the Human Speech Signals, Workshop on Auto. Speech Recognition and Understanding, (2001)

13. Picard, R. W. "Affective Computing". MIT Technical Report \#321, 1995

14. Aggarwal, J. K. \& Cai, Q. Human Motion Analysis: A Review, Computer Vision and Image Understanding, Vol. 73, No. 3, (1999).

15. Msanjila, S.S. \& Afsarmanesh, H. A Multi-Model Approach to Analyze Inter-organizational Trust. In Collaborative Networks reference modeling. ISB N: 978-0-387-79425-9, Springer, New York, Pg 195-216. (2008).

16. Abraham,A., Das, S., \& Roy, S., Swarm Intelligence Algorithms for Data Clustering, Soft Computing for Knowledge Discovery and Data Mining, Oded Maimon and Lior Rokach (Eds.), Springer Verlag, Germany, ISBN 978-0387-69934-9, pp. 279-313, 2007.

17. Salles, P \& Bredeweg. B. Modelling Population and Community Dynamics with Qualitative Reasoning. Ecological Modelling, Volume 195, Issues 1-2, pages 114-128, (2006).

18. Ge, Y., Yang, J.B., Proudlove, N. \& Spring, M. System dynamics modeling for supply-chain management: A case study on a supermarket chain in the UK. In the International Transactions in Operational Research, Vol. 11, pg. 495-509, (2004).

19. Greenland S., \& Brumback, B. An overview of relations among causal modeling methods. In international journal of epidemiology. ISBN: 31-10301037, (2002).

20. Katrenko, S. \& Adriaans, P. Learning Relations from Biomedical Corpora Using Dependency Trees. Invited paper in KDECB (Knowledge Discovery and Emergent Complexity in BioInformatics), Lecture Notes in Bioinformatics. LNBI, vol. 4366, (2006).

21. Methods and Tools for Collaborative Networked Organizations (L.M. Camarinha-Matos, H. Afsarmanesh, M. Ollus- Editors), ISBN: 978-0-38779423-5, Springer, New York, 2008. 